\title{
Motion Control of Small Autonomous Underwater Vehicle in Presence of Parameters Uncertainties
}

\author{
JERZY GARUS ${ }^{1}$, MARIUSZ GIERGIEL ${ }^{2}$ \\ ${ }^{1}$ Faculty of Mechanical and Electrical Engineering \\ Polish Naval Academy \\ 81-103 Gdynia, Smidowicza 69, \\ POLAND \\ ${ }^{2}$ Faculty of Mechanical Engineering and Robotics \\ AGH University of Science and Technology \\ 30-059 Krakow, Al. Mickiewicza 30 \\ POLAND
}

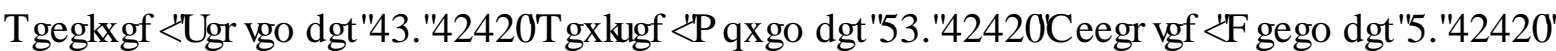

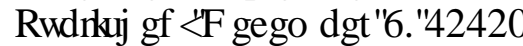

\begin{abstract}
A dynamic model of the underwater vehicle is usually established with parameters uncertainties due to the non-linear and timevarying nature of hydrodynamic forces from the surrounding fluid and external environmental disturbances. The paper investigates the motion control problem of the vehicle in tridimensional space based on model reference adaptive control. A developed autopilot consists of three independent controllers with a parameter adaptation law implemented. A control performance is guaranteed by suitably choosing design parameters. The effectiveness and robustness of the proposed control scheme for trajectory tracking in surge, depth and yaw dynamics is tested through simulations studies.
\end{abstract}

Key-Words: - AUV, motion control, adaptive control, trajectory tracking

\section{INTRODUCTION}

Unmanned underwater vehicles (UUVs) have been a growing research area with strong support from both civilian and military applications. Main benefits of usage of them is to reduce risk to human life under dangerous environment and cost of exploration of deep seas.

The UUVs are generally classified into two main categories: Remotely Operated Vehicles (ROVs), a cubic-shaped submersibles physically connected to the surface by a theter cable and requiring instructions from a human pilot and Autonomous Underwater Vehicles (AUVs), a torpedo-shaped ones which operate with no physical link with the surface and executing a mission without human interventions. Nowadays, the AUVs have captured increasing attention due to their wide area of usage and advantageous features.

Control problem of the UUVs have been considered extensively in last decades, and many different algorithms are proposed in literature to achieve the desired behavior of these vehicles, including: PID control, robust control, adaptive control, sliding mode control, back-stepping control, predictive control, fuzzy control, neural network control, and other hybrid control methods $[1,2,6,7,9,12,14,15,16,17]$.

Control of the AUV with taking into consideration all real conditions of operation is a complex process, mainly come from parametric uncertainties caused by poor knowledge of hydrodynamic coefficients and unpredictable 
external disturbances in terms of waves and sea currents. Moreover, its dynamics can change according to the alteration of configuration to be suited to a mission. Therefore, adaptive control approach is widely used in underwater community to deal with uncertainties in constant or slow varying parameters.

In this paper, based on Model Reference Adaptive Control (MRAC) technique, a controller/autopilot for trajectory tracking of the AUV in underwater space is developed. An idea of the MRAC is based on forcing the control object to follow a reference model, i.e. an adaptive controller has to decrease to zero an error between parameters of the reference model and real object $[5,10,11$, 14]. The controller is parameterized and provides tracking whereas an adaptation mechanism is used to adjust parameters in a control law. Numerical simulations are performed to show the effectiveness of the proposed control scheme.
The work consists of five sections. After the introduction, a brief description of equations of motion of the AUV is provided. Then a nonlinear adaptive control law is described. Next section presents results of simulation study. Conclusions are given in the last section.

\section{EQUATIONS OF MOTION OF AUV}

Underwater vehicles move in all three dimensions and six degrees of freedom. A mathematical model of UUV motion process in viscous fluid is introduced by system of differential equations of motion. These equations involves two coordinate frames of reference: the body-fixed frame $\left(\mathrm{O}_{0} \mathrm{X}_{0} \mathrm{Y}_{0} \mathrm{Z}_{0}\right)$ and the earth-fixed frame (OXYZ) (see Fig. 1).

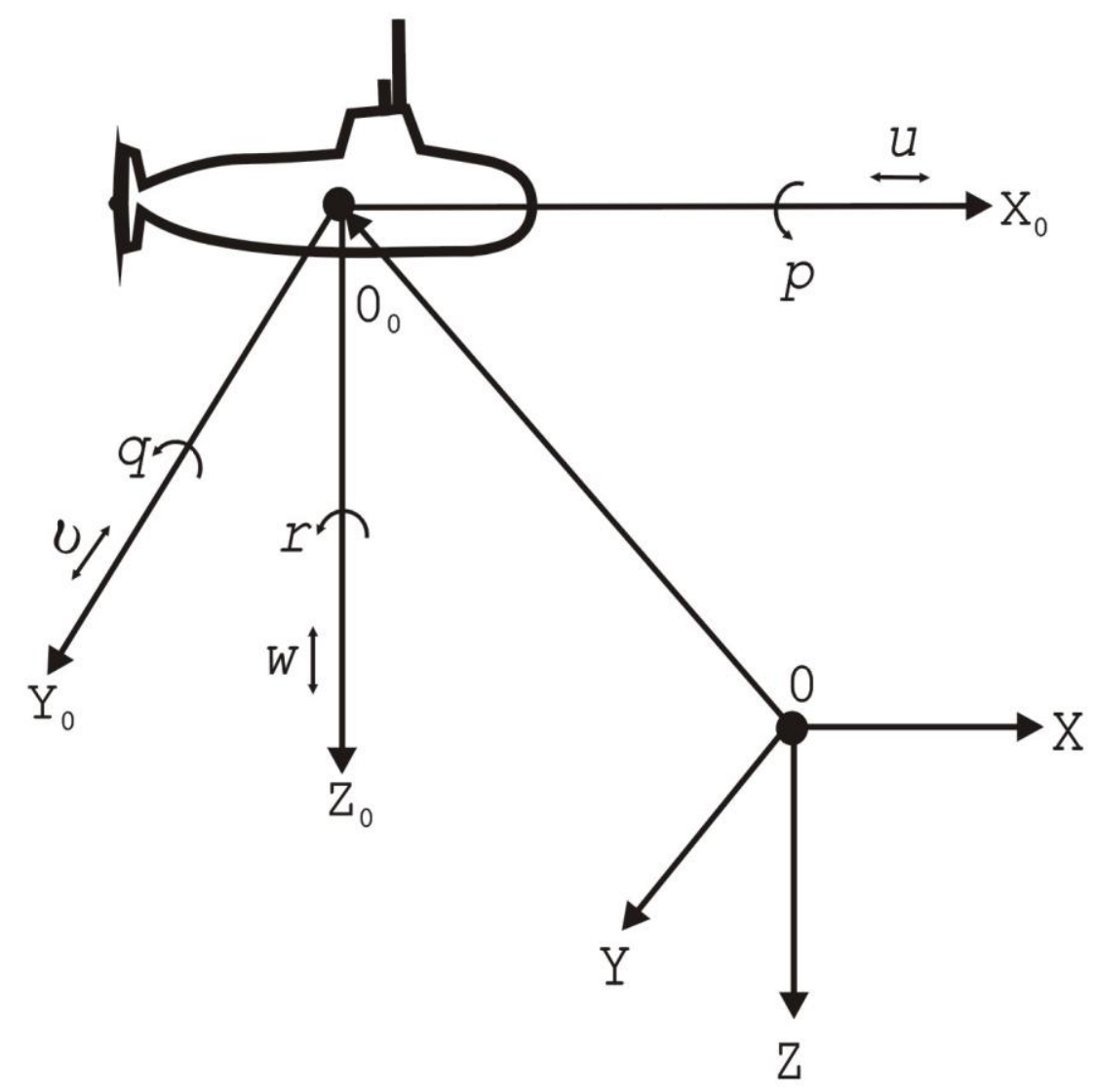

Fig. 1. The UUV with coordinate frames of reference. 
General motion of the AUV describes the following vectors $[1,3]$ :

$$
\begin{aligned}
\boldsymbol{\eta} & =[x, y, z, \phi, \theta, \psi]^{T} \\
\mathbf{v} & =[u, v, w, p, q, r]^{T} \\
\boldsymbol{\tau} & =[X, Y, Z, K, M, N]^{T}
\end{aligned}
$$

where:

$$
\begin{aligned}
& \boldsymbol{\eta} \text { - vector of position and orientation } \\
& \text { in the earth-fixed frame; } \\
& x, y, z \text { - coordinates of position; } \\
& \phi, \theta, \psi \text {-coordinates of orientation (Euler } \\
& \text { angles); } \\
& \mathbf{v}-\text { vector of linear and angular velocities } \\
& \text { in the body-fixed frame; } \\
& u, v, w \text { - linear velocities along longitudinal, } \\
& \text { transversal and vertical axes; } \\
& p, q, r \text { - angular velocities about longitudinal, } \\
& \text { transversal and vertical axes; } \\
& \tau \quad-\text { vector of forces and moments acting } \\
& \text { on the vehicle in the body-fixed } \\
& \text { frame; } \\
& X, Y, Z \text { - forces along longitudinal, transversal } \\
& \text { and vertical axes; } \\
& K, M, N \text { - moments about longitudinal, } \\
& \text { transversal and vertical axes. }
\end{aligned}
$$

Considering the generalized inertial forces, the hydrodynamic effects, the gravity and buoyancy contributions as well as the forces of the actuators (i.e. thrusters), a dynamic model of the autonomous underwater vehicle in matrix form can be written as follows $[2,6]$ :

$$
\mathbf{M} \dot{\mathbf{v}}+\mathbf{C}(\mathbf{v}) \mathbf{v}+\mathbf{D}(\mathbf{v}) \mathbf{v}+\mathbf{g}(\boldsymbol{\eta})=\boldsymbol{\tau}
$$

where:

$$
\begin{array}{ll}
\mathbf{M} & - \text { inertia matrix (including added } \\
& \text { mass); } \\
\mathbf{C}(\mathbf{v}) & - \text { matrix of Coriolis and centripetal } \\
& \text { terms (including added mass); } \\
\mathbf{D}(\mathbf{v}) \quad- & \text { hydrodynamic damping and lift } \\
& \text { matrix; } \\
\mathbf{g}(\boldsymbol{\eta}) \quad- & \text { vector of gravitational forces and } \\
& \text { moments. }
\end{array}
$$

\section{III.CONTROLLER DESIGN}

For the AUVs there are parametric uncertainties in the mathematical model (2), and certain parameters are generally unknown. Hence, parameter estimation is necessary in case of the MRAC. For this purpose it is assumed that the equations of motion (2) are linear according to a parameter vector $\mathbf{p}[2,13]$ :

$$
\mathbf{M} \dot{\mathbf{v}}+\mathbf{C}(\mathbf{v}) \mathbf{v}+\mathbf{D}(\mathbf{v}) \mathbf{v}+\mathbf{g}(\boldsymbol{\eta}) \cong \mathbf{Y}(\boldsymbol{\eta}, \mathbf{v}, \dot{\mathbf{v}}) \mathbf{p}=\boldsymbol{\tau}
$$

where $\mathbf{Y}(\boldsymbol{\eta}, \mathbf{v}, \dot{\mathbf{v}})$ is a known matrix function of measured signals usually referred as the regressor matrix and $\mathbf{p}$ is a vector of uncertain or unknown parameters.

Under assumption that parameters of desired trajectory $\boldsymbol{\eta}_{d}$ and $\mathbf{v}_{d}$ are given, vectors $\boldsymbol{\eta}$ and $\mathbf{v}$ measured, and $\hat{\mathbf{M}}, \hat{\mathbf{C}}(\mathbf{v}), \hat{\mathbf{D}}(\mathbf{v}), \hat{\mathbf{g}}(\boldsymbol{\eta})$ and $\hat{\mathbf{p}}$ are estimates of matrices and vectors $\mathbf{M}, \mathbf{C}(\mathbf{v}), \mathbf{D}(\mathbf{v})$, $\mathbf{g}(\boldsymbol{\eta})$ and $\mathbf{p}$, the following nonlinear control law can be applied $[8,10]$ :

$$
\begin{aligned}
\boldsymbol{\tau} & =\hat{\mathbf{M}} \dot{\mathbf{u}}+\hat{\mathbf{C}}(\mathbf{v}) \mathbf{u}+\hat{\mathbf{D}}(\mathbf{v}) \mathbf{u}+\hat{\mathbf{g}}(\boldsymbol{\eta})-\mathbf{K}_{D} \mathbf{s}= \\
& =\mathbf{Y}(\boldsymbol{\eta}, \mathbf{v}, \mathbf{u}, \dot{\mathbf{u}}) \hat{\mathbf{p}}-\mathbf{K}_{D} \mathbf{S}
\end{aligned}
$$

where:

$$
\begin{aligned}
& \mathbf{u}=\mathbf{v}_{d}-\boldsymbol{\Lambda} \mathbf{e}_{1} \\
& \mathbf{e}_{1}=\boldsymbol{\eta}-\boldsymbol{\eta}_{d} \\
& \boldsymbol{\Lambda} \text { - positive definite weighting matrix; } \\
& \mathbf{K}_{D} \text { - positive definite diagonal matrix; } \\
& \mathbf{s}=\mathbf{e}_{2}+\boldsymbol{\Lambda} \mathbf{e}_{1} \\
& \mathbf{e}_{2}=\mathbf{v}-\mathbf{v}_{d}
\end{aligned}
$$

Choosing the parameter update law as $[8,13]$ :

$$
\dot{\hat{\mathbf{p}}}=-\Gamma \mathbf{Y}^{T}(\boldsymbol{\eta}, \mathbf{u}, \dot{\mathbf{u}}) \mathbf{s}
$$

where $\Gamma$ is a positive definite symmetric matrix, stability of the control system and convergence $\mathbf{s}$ to zero is guaranteed.

A block scheme of the described adaptive control law shows Fig. 2. 


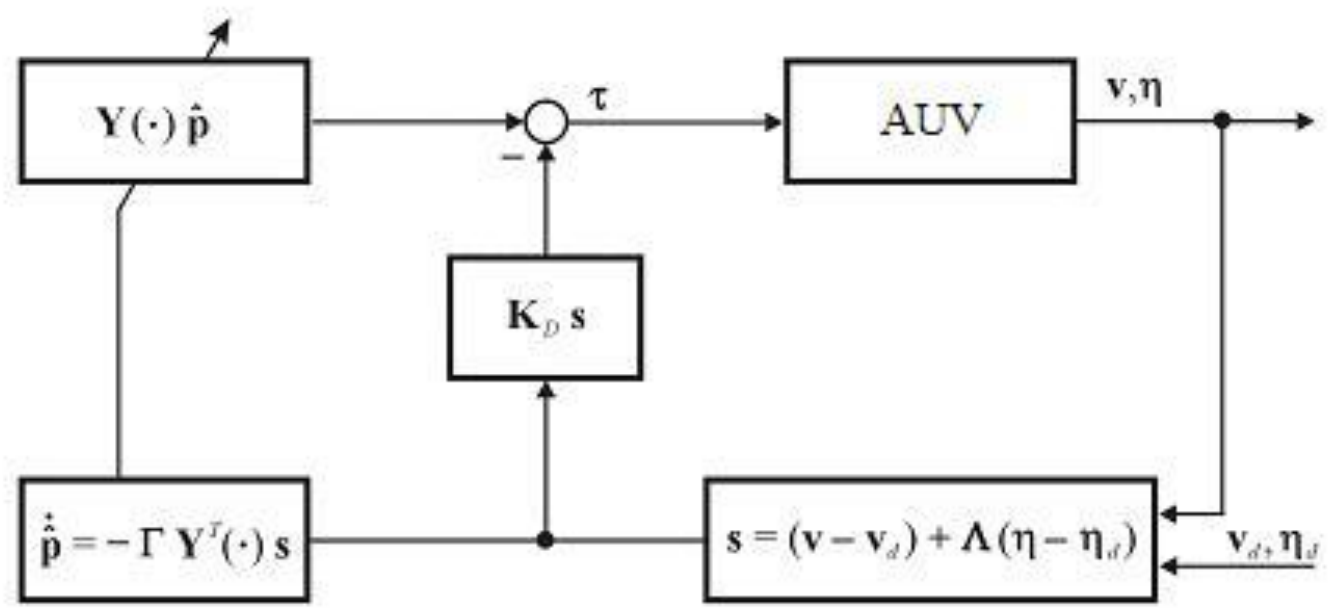

Fig. 2. The block diagram of the parameter adaptation law.

\section{SIMULATION STUDY}

A main task of the proposed tracking control system is to minimize distance of attitude of a vehicle's centre of gravity to the desired trajectory under assumptions:

1. the vehicle can move with varying linear velocities $u, w$ and angular velocity $r$;

2. velocities $u, w, r$ and coordinates of position $x$, $y, z$ and heading $\psi$ are measurable;

3. the desired trajectory is given by means of set of way-points $\left\{\left(x_{d i}, y_{d i}, z_{d i}\right)\right\}$;

4. the reference trajectory between two successive way-points is defined as a smooth and bounded curve;

5. the command signal $\tau$, calculated from the control law (4), consists of three components: $\tau_{1}=\tau_{X}=X, \tau_{2}=\tau_{Z}=Z \quad \tau_{3}=\tau_{N}=N$

A structure of the control system is depicted in Fig. 3.

To validate the performance of the proposed adaptive control law some simulations in the MATLAB/Simulink environment have been conducted. A mathematical model of the vehicle is dedicated to a real construction called Gannet [4], designed and built for the Polish Navy (see Fig. 4). The AUV is a torpedo shaped vehicle, $1400 \mathrm{~mm}$ long, $50 \mathrm{~kg}$ mass and capable of reach a maximum depth of $100 \mathrm{~m}$. It is controllable in four degrees of freedom (DOFs) and equipped with a propulsion system consisting of five thrusters. Four of them are installed in the stern and assure surge, pitch and yaw motions. The fifth one, located in a middle of the body, is responsible for heave motion. All parameters of the underwater vehicle's dynamics used in a simulation study are given in the Appendix A. 


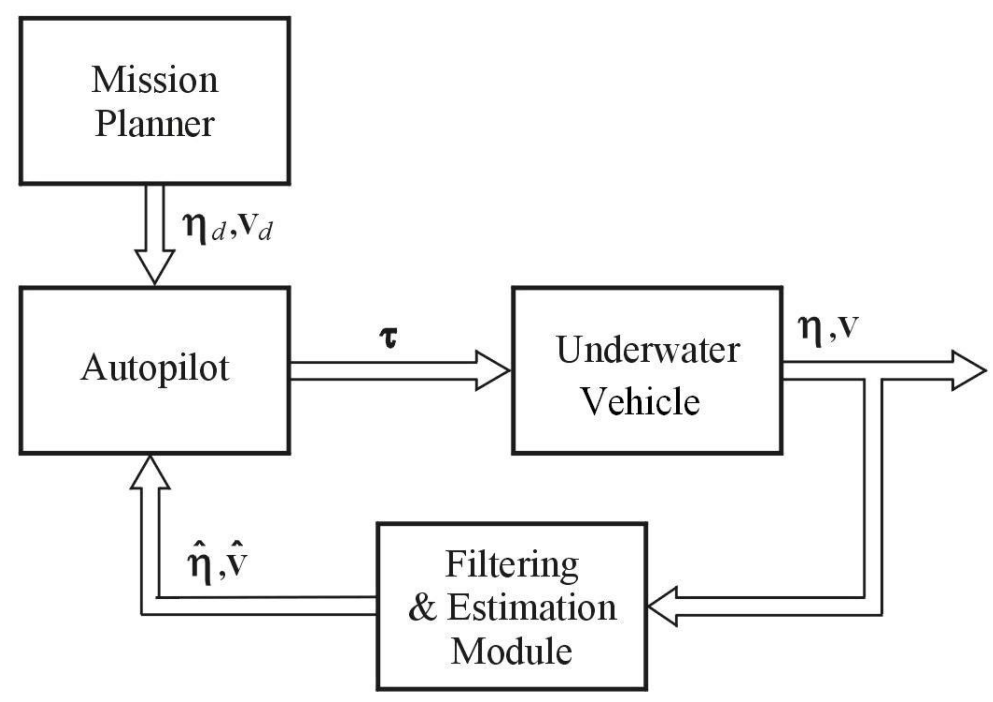

Fig. 3. The structure of the control system.

Numerical experiments were made under the following assumptions:

1. the vehicle has to follow the desired trajectory staring from $(0 \mathrm{~m}, 0 \mathrm{~m},-5 \mathrm{~m})$, passing waypoints: $(0 \mathrm{~m}, 0 \mathrm{~m},-10 \mathrm{~m}),(50 \mathrm{~m}, 0 \mathrm{~m},-10 \mathrm{~m})$, (80 m, $30 \mathrm{~m},-10 \mathrm{~m}), \quad(80 \mathrm{~m}, 80 \mathrm{~m},-10 \mathrm{~m})$, $(30 \mathrm{~m}, 80 \mathrm{~m},-10 \mathrm{~m}), \quad(10 \mathrm{~m}, 50 \mathrm{~m},-10 \mathrm{~m})$, $(10 \mathrm{~m},-10 \mathrm{~m},-10 \mathrm{~m})$, and ending at $(10 \mathrm{~m}$, $10 \mathrm{~m},-15 \mathrm{~m})$;

2. the way-point is reached whether the vehicle is inside of a meter circle of acceptance. 


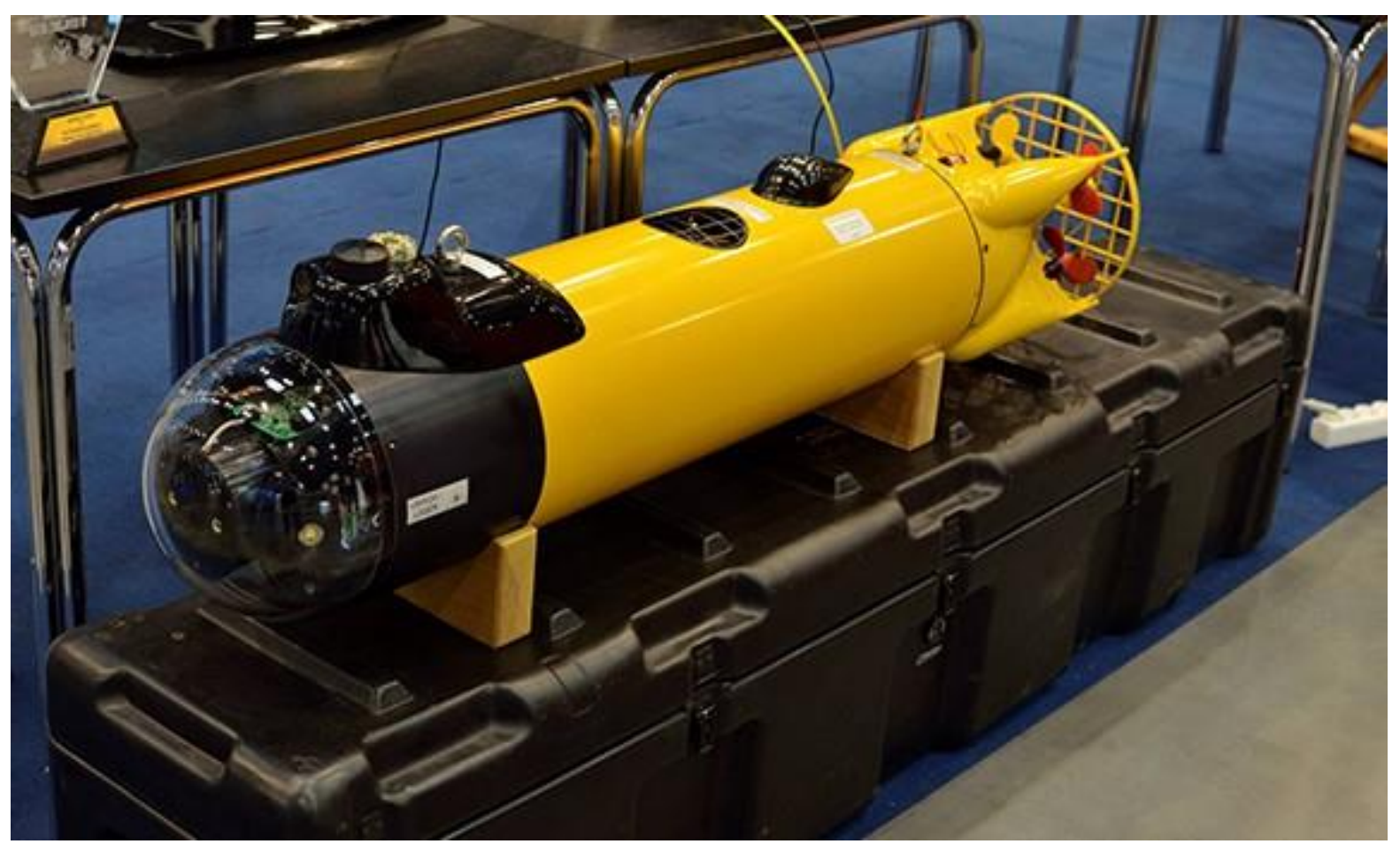

Fig. 4. The view of the AUV.

Time-varying reference trajectories at the waypoint $i$ to the next way-point $i+1$ were generated using predefined speed profiles [5, 13]. Such approach allows keeping constant speed along certain part of the trajectory. For above assumptions and initial conditions:

$$
\begin{array}{ll}
\eta_{d k}\left(t_{b}\right)=\eta_{0}, & \dot{\eta}_{d k}\left(t_{b}\right)=\dot{\eta}_{0} \\
\eta_{d k}\left(t_{f}\right)=\eta_{1}, & \dot{\eta}_{d k}\left(t_{f}\right)=\dot{\eta}_{1} \\
\max \dot{\eta}_{d k}(t)=\dot{\eta}_{\max } &
\end{array}
$$

where $k=\overline{1,3}$, the $k^{\text {th }}$ segment of the trajectory in a period of time $t \in\left\langle t_{b}, t_{f}\right\rangle$ was modelled according to the expression:

$$
\eta_{d k}(t)= \begin{cases}\frac{\eta_{0}+\frac{\dot{\eta}_{\max }-\dot{\eta}_{0}}{2 t_{m}} t^{2}}{\eta_{1}+\eta_{0}-\dot{\eta}_{\max }\left(t_{f}-2 t_{m}\right)} & t_{b} \leq t \leq t_{m} \\ +\dot{\eta}_{\max }\left(t-t_{m}\right) & t_{m}<t \leq t_{f}-t_{m} \\ \eta_{1}-\frac{\dot{\eta}_{\max }-\dot{\eta}_{1}}{2 t_{m}}\left(t_{f}-t\right)^{2} & t_{f}-t_{m}<t \leq t_{f}\end{cases}
$$

where $\quad t_{m}=t_{f}-\frac{\eta_{1}-\eta_{0}}{\dot{\eta}_{\max }}$.
The algorithm with adaptive control law was worked out basis on a simplified AUV model proposed in [2]:

$$
\mathbf{M}_{d} \dot{\mathbf{v}}+\mathbf{D}_{d}(\mathbf{v}) \mathbf{v}=\boldsymbol{\tau}
$$

where all dynamical cross-coupling terms are neglected. Here $\mathbf{M}_{d}$ and $\mathbf{D}_{d}(\mathbf{v})$ are diagonal matrices with the diagonal elements of the inertia and damping matrices, consequently. Uncertainties in the above model are compensated by the developed control system.

The model (6) for motion of 3 DOFs takes a form:

$$
\begin{aligned}
& m_{X} \dot{u}+d_{X}|u| u=\tau_{X} \\
& m_{Z} \dot{w}+d_{Z}|w| w=\tau_{Z} \\
& m_{N} \dot{r}+d_{N}|r| r=\tau_{N}
\end{aligned}
$$

Define the parameter vector $\mathbf{p}$ in a form $\mathbf{p}=\left[\begin{array}{llllll}m_{X} & d_{X} & m_{Z} & d_{Z} & m_{N} & d_{N}\end{array}\right]^{T}$ the expression (6) can be written as:

$$
\mathbf{Y}(\mathbf{v}, \dot{\mathbf{v}}) \mathbf{p}=\boldsymbol{\tau}
$$

where: 


$$
\mathbf{Y}(\mathbf{v}, \dot{\mathbf{v}})=\left[\begin{array}{cccccc}
\dot{u} & |u| u & 0 & 0 & 0 & 0 \\
0 & 0 & \dot{w} & |w| w & 0 & 0 \\
0 & 0 & 0 & 0 & \dot{r} & |r| r
\end{array}\right]
$$

Results of track-keeping are presented in Fig. 5 and Fig. 6. They show that the designed autopilot enhanced quite good tracking control of the desired trajectory in the underwater space. The position of the AUV in 3D converges to the reference position.
The change of control inputs for uncertain parameters are demonstrated in Figure 7.

During simulations it was assumed that the true values of components of the vector $\mathbf{p}$ are unknown. An evaluation process started from certain values, which were equal to half of nominal values of mass and damping coefficients. Time histories of estimated parameters during tracking are presented in Fig. 8.

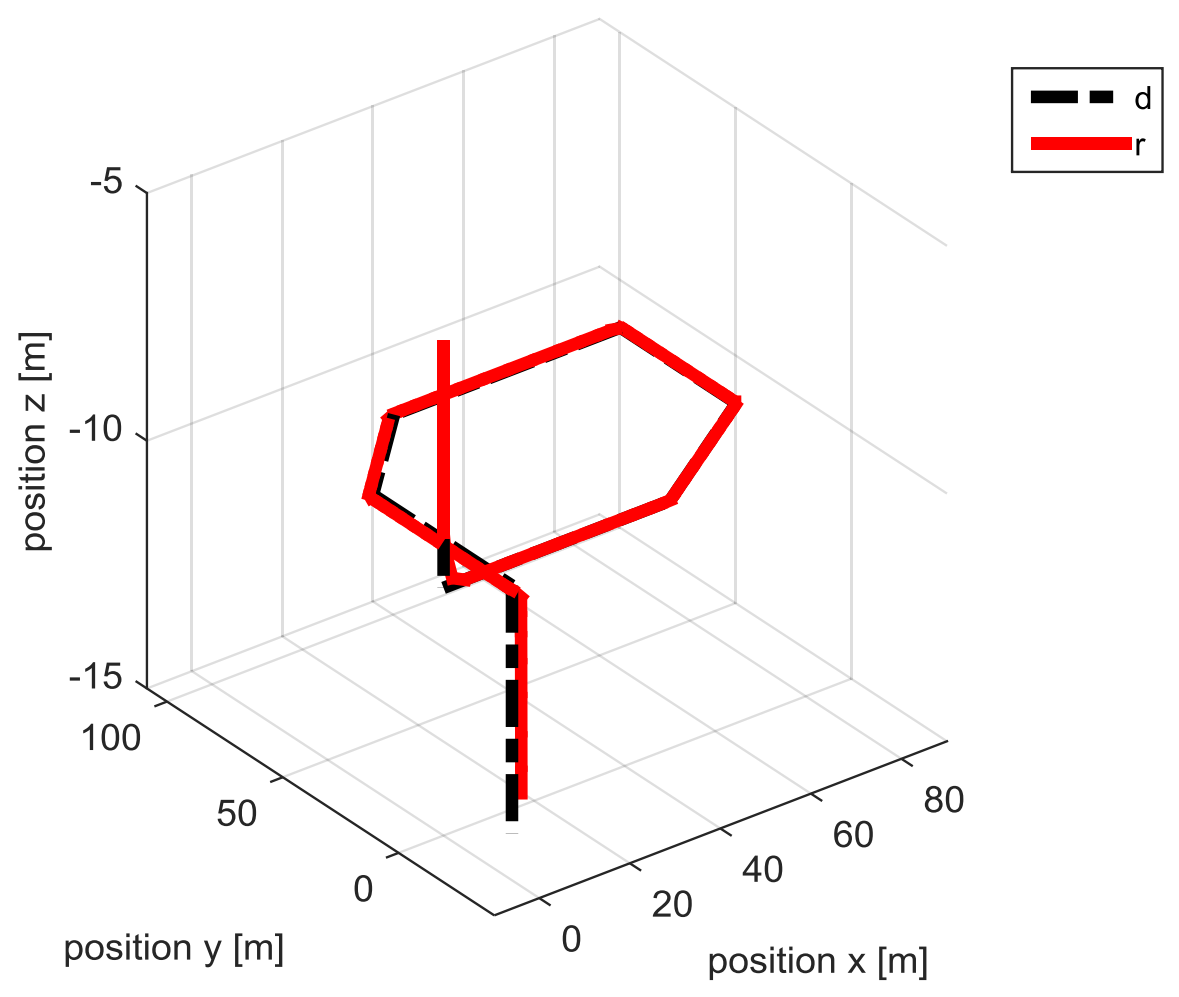

Fig. 5. Motion of the AUV in three-dimensional space: (d) desired and (r) realized trajectories. 

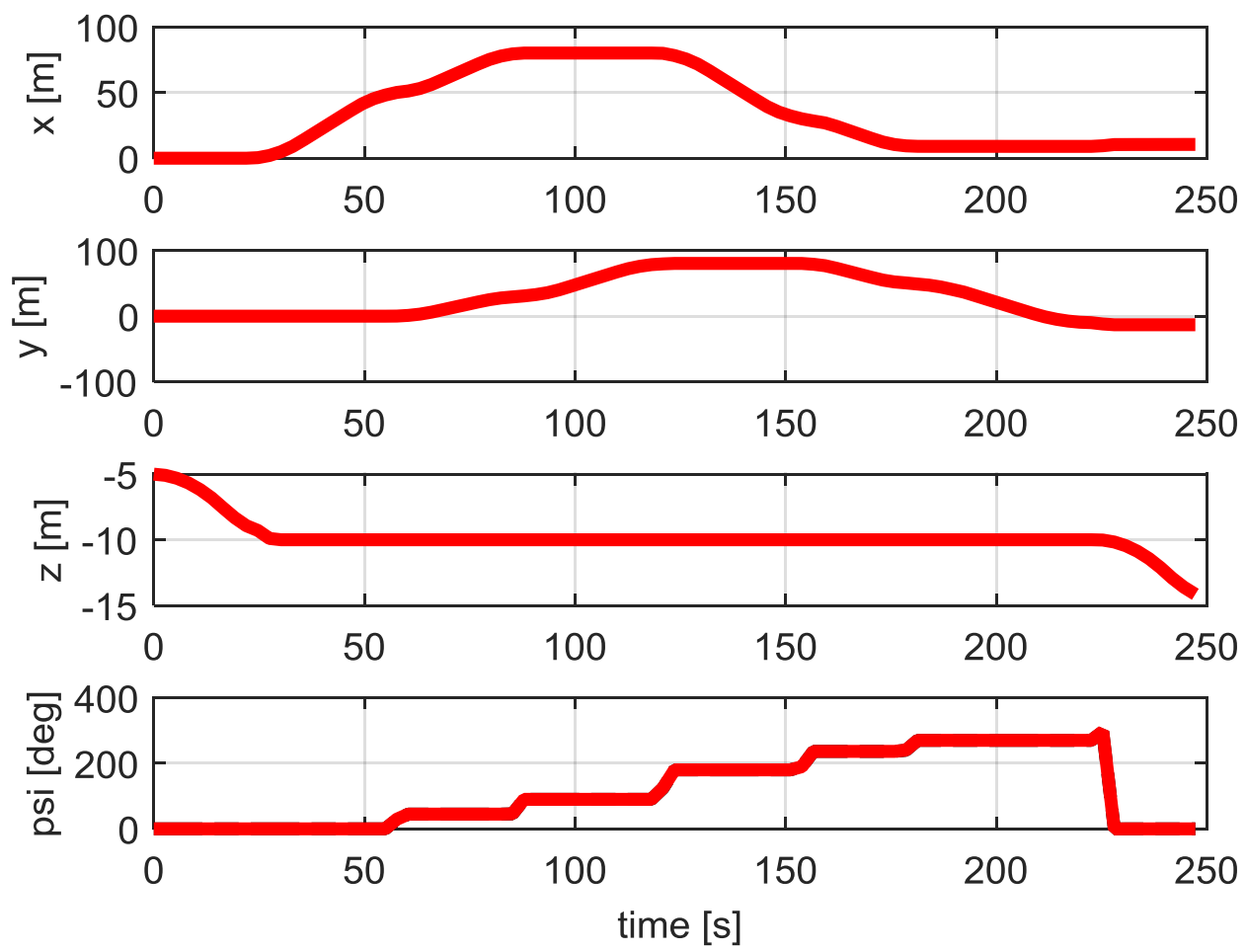

Fig. 6. Changes of vehicle's positions $x, y, z$ and heading $\psi$, when performing the experiment in Figure 5.

The results of simulations show that the designed autopilot enhanced quite good tracking control of the desired trajectory in the underwater space. The position of the AUV converges to the reference position (Fig. 5). It is obtained, although the estimated mass and damping coefficients in 3 DOFs motion generally differ to the real ones. It can be assumed that a better estimation process should improve control quality. Therefore, this problem needs further investigations. 

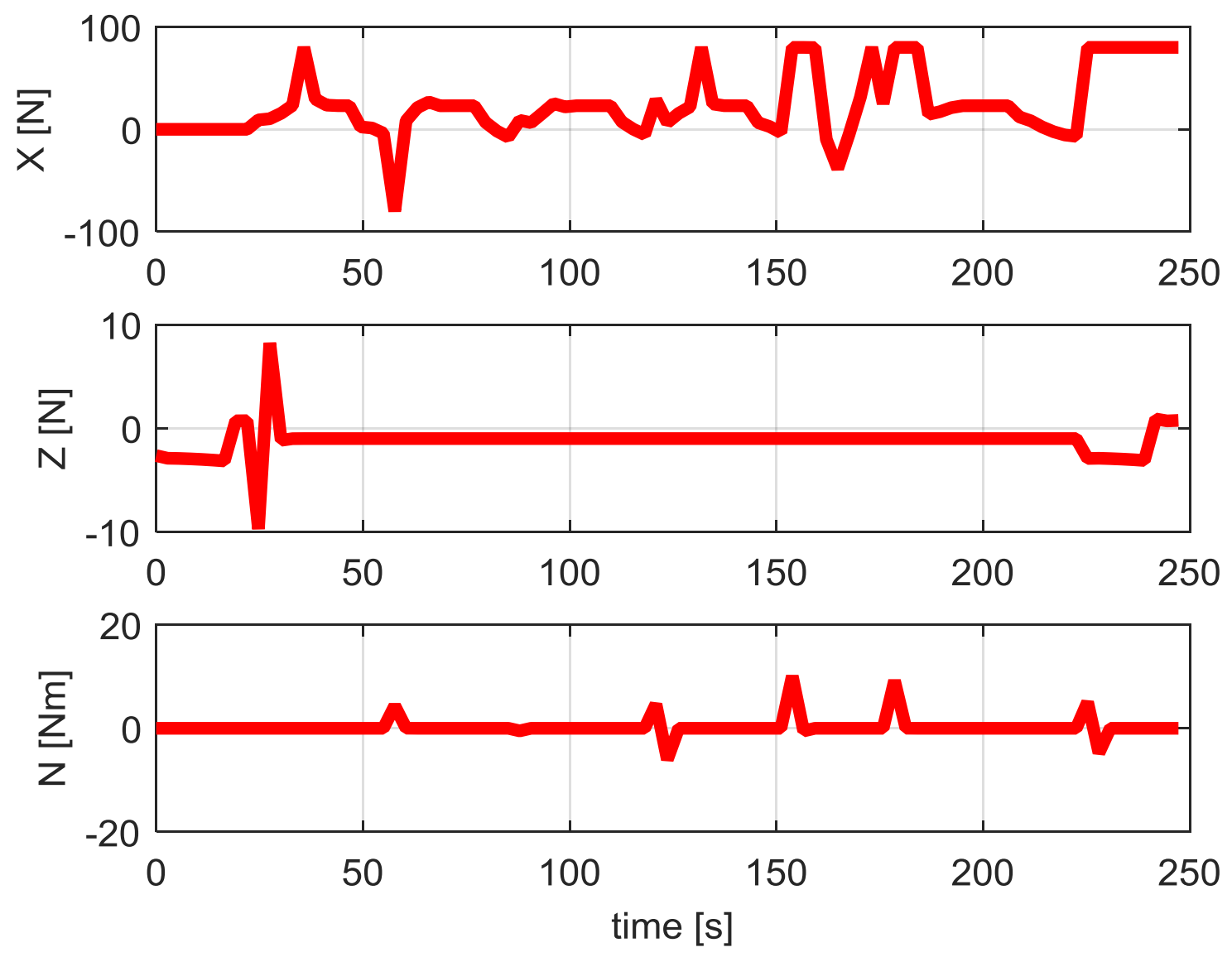

Fig. 7. Changes of command signals, when performing the experiment in Figure 5. 

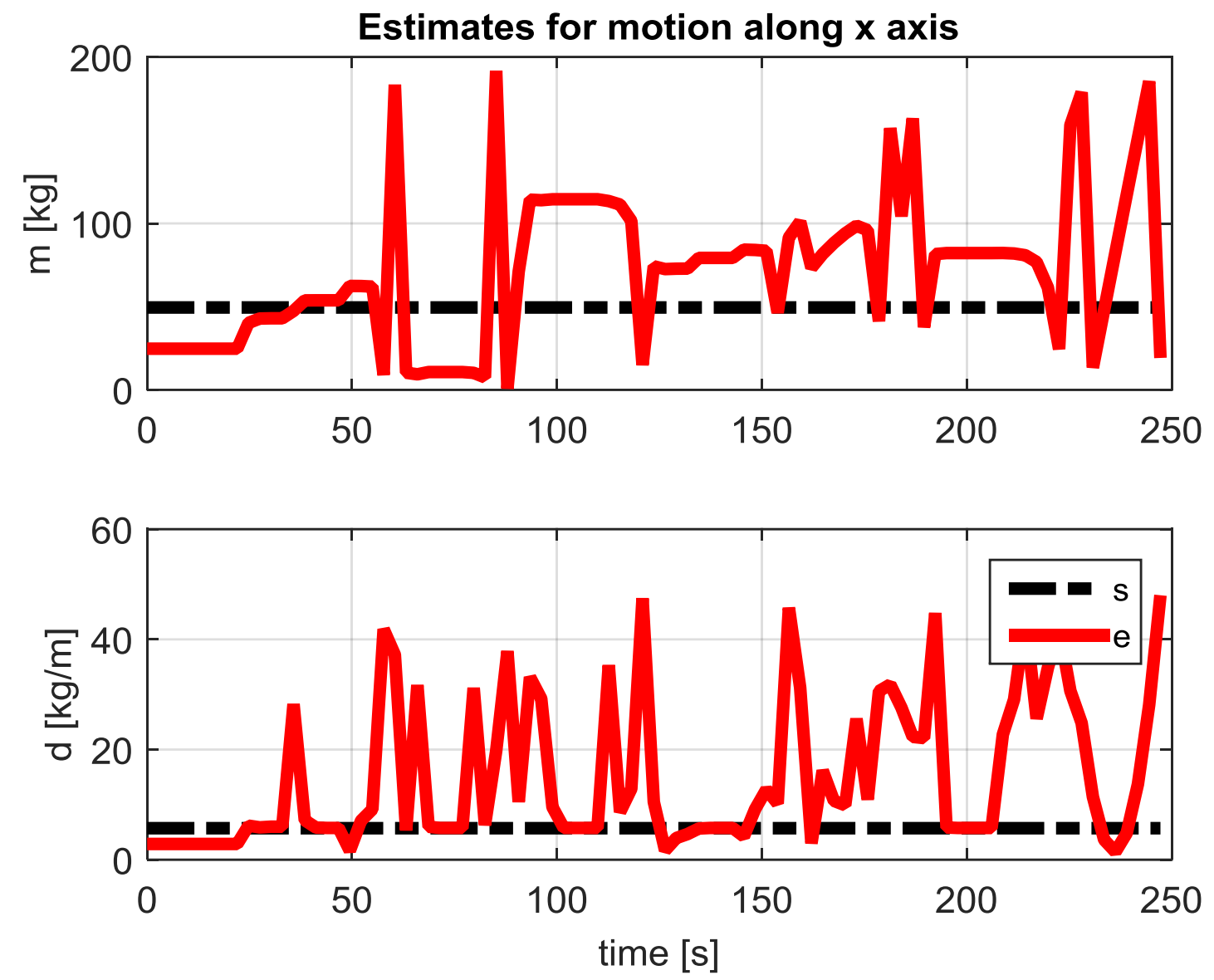

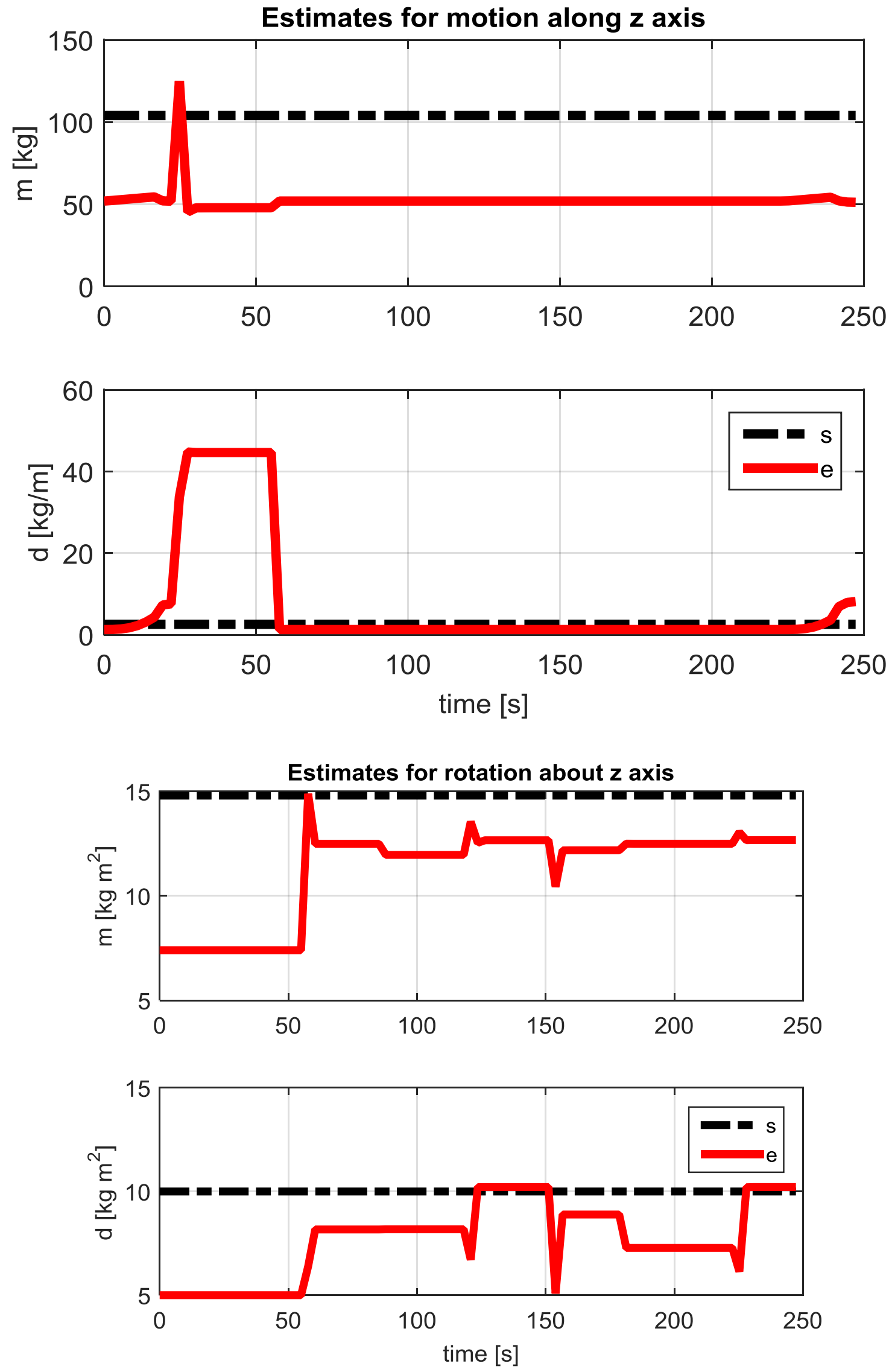

Fig. 8. Evaluations of mass and damping coefficients: (s) set and (e) estimate values. 


\section{CONCLUSIONS}

In the paper an application of the model reference adaptive control to steering the autonomous underwater vehicle along a predefined trajectory was presented. Surge, depth and yaw dynamics have been considered separately and adaptive controllers have been designed independently for any of them.

Simulations study implemented taking into account the model uncertainties have shown the successive performance of the proposed control scheme.

Further work is devoted to real-world testing the effectiveness and robustness of the proposed control law towards external disturbances and persistent parametric uncertainties. 
References:

[1] T. I. Fossen, Guidance and Control of Ocean Vehicles, John Wiley and Sons, Chichester, 1994.

[2] T. I. Fossen, Marine Control Systems, Marine Cybernetics AS, Trondheim 2002.

[3] T. I. Fossen, Handbook of Marine Craft Hydrodynamics and Motion Control, John Wiley and Sons, Chichester, 2011.

[4] J. Garus, Simulation of Spatial Motion of Selfpropelled Mine Counter Charge. WSEAS Transactions on Systems and Control, Vol. 1, 2006, pp. 138-142.

[5] J. Garus, Model Reference Adaptive Control of Underwater Robot in Spatial Motion. In Modelling Dynamics in Process and Systems, W. Mitkowski and J. Kacprzyk (Eds.), Springer-Verlag, Berlin Heildelberg 2009, pp. $71-83$

[6] J. Garus, R. Studanski, T. Batur, Passive Fault Tolerant Control Allocation for Small Unmanned Underwater Vehicle, Journal of Marine Engineering and Technology, Vol. 16, No. 4, 2017, pp. 420-423.

[7] J. Giergiel, K. Kurc, M. Giergiel, Mechatronics Designing of Inspection Robots, Rzeszow University of Technology Press, Rzeszow, 2010 (in Polish).

[8] B. Lantos, L. Muton, Nonlinear Control of Vehicles and Robots, Springer-Verlag, London, 2011.

[9] L. Lapierre, J. Bruno, Robust Nonlinear PathFollowing Control of a AUV, IEEE Journal of Oceanic Engineering, Vol. 33, No. 2, 2008, pp. 89-102.

[10] K. Ogata, Modern Control Engineering, Prentice Hall, New Jersey, 2002.

[11] J. Rothe, J. Zevering, M. Strohmeierand, S. Montenegro, A Modified Model Reference Adaptive Controller (M-MRAC) Using an Updated MIT-Rule for the Altitude of a UAV, Electronics, Vol. 9, No. 7, 2020, pp. 583-591.

[12] C. Silvestre, A. Pascoal, Control of the Infante AUV Using Gain Scheduled Static Output Feedback, Control Engineering Practice, Vol. 12, No. 12, 2004, pp. 1501-1509.

[13] M. W. Spong, M. Vidyasagar, Robot Dynamics and Control, John Wiley and Sons, Chichester, 1989.

[14] Y. C. Sun, C. C. Chea, Adaptive Control Schemes for Autonomous Underwater Vehicle, Robotica, Vol. 27, No 1, 2009, pp. 119-129.

[15] P. Szymak, J. Malecki, Control System of Underwater Vehicle Based on Artificial
Intelligence Methods, Automation and Robotics, Vol. 17, 2008, pp. 285-296.

[16] X. Xiang, C. Yu, L. Lapierre, J. Zhang, Q. Zhang, Survey on Fuzzy-Logic Based Guidance and Control of Marine Surface Vehicles and Underwater Vehicles, International Journal of Fuzzy Systems, Vol. 20, 2018, pp. 572-586.

[17] J. Yuh, Design and Control of Autonomous Underwater Robots: A Survey. Autonomous Robots, Vol. 8, No 1, 2000, pp. 7-24. 


\section{Appendix A}

Parameters of the AUV's mathematical model utilized in numerical experiments:

$\mathbf{M}=\operatorname{diag}\left\{\begin{array}{llllll}49.5 & 104.0 & 104.0 & 0.8 & 18.9 & 18.9\end{array}\right\}$

$\mathbf{D}(\mathbf{v})=\mathbf{D}+\mathbf{D}_{d}(\mathbf{v})$

where:

$\mathbf{D}=\operatorname{diag}\left\{\begin{array}{llllll}0.09 & 0.19 & 0.19 & 0.0 & 0.8 & 0.7\end{array}\right\} ;$

$\mathbf{D}_{d}(\mathbf{v})=\operatorname{diag}\left\{\begin{array}{ccc}5.92|u| & 2.58|v| & 2.58|w| \\ 0 & 11.0|q| & 10.0|r|\end{array}\right\}$

$\mathbf{g}(\boldsymbol{\eta})=\left[\begin{array}{c}-\sin (\theta) \\ -\cos (\theta) \sin (\phi) \\ \cos (\theta) \cos (\phi) \\ 0 \\ 0 \\ 0\end{array}\right]$

$\mathbf{C}(\mathbf{v})=\mathbf{0}$.

Values of the matrices applied in adaptive control law (4):

$$
\begin{aligned}
& \mathbf{K}_{D}=\operatorname{diag}\left\{\begin{array}{llllll}
50.0 & 0.0 & 40.0 & 0.0 & 0.0 & 20.0
\end{array}\right\} \\
& \boldsymbol{\Gamma}=\operatorname{diag}\left\{\begin{array}{llllll}
0.01 & 0.0 & 0.005 & 0.0 & 0.0 & 0.005
\end{array} ;\right. \\
& \boldsymbol{\Lambda}=\operatorname{diag}\left\{\begin{array}{llllll}
2.0 & 0.0 & 20.0 & 0.0 & 0.0 & 5.0
\end{array}\right\}
\end{aligned}
$$




\section{Creative Commons Attribution License 4.0}

(Attribution 4.0 International, CC BY 4.0)

This article is published under the terms of the Creative Commons Attribution License 4.0

https://creativecommons.org/licenses/by/4.0/deed.e n_US 